\title{
Health Support System for Diabetes Prevention using Networked Health-monitoring Equipment
}

\author{
Masashi Sugano, Masahide Imaki, Yukie Yoshida \\ School of Comprehensive Rehabilitation, \\ Osaka Prefecture University \\ 3-7-30 Habikino, Habikino-shi, Osaka, Japan \\ Email: sugano@rehab.osakafu-u.ac.jp
}

\author{
Nobuyoshi Ando \\ Business Incubation Division, \\ Hitachi Ltd. \\ 1-6-6 Marunouchi, Chiyoda-ku, Tokyo, Japan \\ Email: nobuyoshi.ando.kb@hitachi.com
}

\begin{abstract}
For participants of diabetes prevention programs, we consider that it is effective to record vital signs, such as weight and blood pressure, and to receive instruction based on such readings during the follow-up period in order to avoid rebound. Although the Internet is an effective medium for recording data and reporting everyday health status, health support systems that require the use of a $\mathrm{PC}$ can place a heavy burden on users, especially elderly users. In this research, we utilized a sensor information web service which is a home network based on ZigBee, and discuss effective implementation of a follow-up system. We explained and demonstrated the proposed system to participants who had completed a diabetes prevention program and administered a questionnaire about their impression of system use. From the questionnaire results, we clarify the considerations and requirements necessary for introducing such a home network system with the elderly.
\end{abstract}

\section{INTRODUCTION}

In 2000, the Japanese government launched the project "Healthy Japan 21" aiming at extending healthy life expectancy and improving quality of life by preventing lifestylerelated diseases. Moreover, a specific medical examination for the prevention of metabolic syndrome accompanied by specific health guidance was also started from April 2008. Among lifestyle-related diseases, diabetes, which has few subjective symptoms, is thought to have a prevalence of one in five adults. Diabetes and other lifestyle-related diseases can be prevented by improving lifestyle, and the development of suitable teaching programs is therefore urgently needed.

Since 2005, Osaka Prefecture University and Habikino city government have run a Diabetes Prevention Program as a joint enterprise. This six-month program provides individual and group counseling covering the three areas of "nutrition", "exercise", and "oral care", all of which are important to help diabetics manage their condition. The program has been confirmed to be effective for increasing muscle bulk, decreasing Hemoglobin A1c (HbAlc) levels, and improving oral hygiene [1-5]. However, once participants have completed the diabetic prevention program, there is currently no follow-up study monitoring any changes to their condition. In general, economic restrictions on human resources mean that such educational programs can be provided in the short term only and the rebound phenomenon is frequently seen among participants after the program ends. It is therefore important that participants continue the lifestyle changes they adopted during the program over as long a period of time as possible [6]. As a means to support participants in continuing such management of their condition from at home, information technologies, such as the personal computer and the Internet, have attracted attention, and research and development of various IT systems is ongoing [7].

Information devices such as personal computers and cellular phones can handle multimedia information not only in text and numeric form but also in image and video form. The use of such devices can be effective in self management because they can be used remotely and at any time. However, many people cannot sufficiently master the use of such devices. Among the middle-aged and elderly, who especially need to be taught about lifestyle-related diseases, there are still barriers, such as cost, to them using such technologies. As such, it cannot be said that any support system developed that requires the direct use of a personal computer or the Internet has high flexibility [8]. Moreover, even among the younger generation who can sufficiently master the Internet, using a system which requires the input of weight and dietary data every day exerts a heavy burden [9].

Given this background, in this study we aimed at developing a system which collects follow-up data of the participant after the diabetes prevention program has ended but which places no burden on the user, in order to perform an evaluation of their progress. We built a home network based on ZigBee [10], and introduced a sensor information web service which can collect information obtained from home sensors via the Internet. In this way, the user need only take measurement data every day using a body composition scale and a sphygmomanometer, and the measurement data is automatically uploaded to a server. We proposed using this system in the follow up of participants who had completed a diabetes prevention program. Therefore, as a pre-implementation step with the aim clarifying the considerations and requirements necessary at the time of introducing such a home network device, we explained and demonstrated the proposed system to the participants and collected their opinions about possible future use by questionnaire.

The remainder of this paper is organized as follows. We present an outline of the diabetes prevention program in 


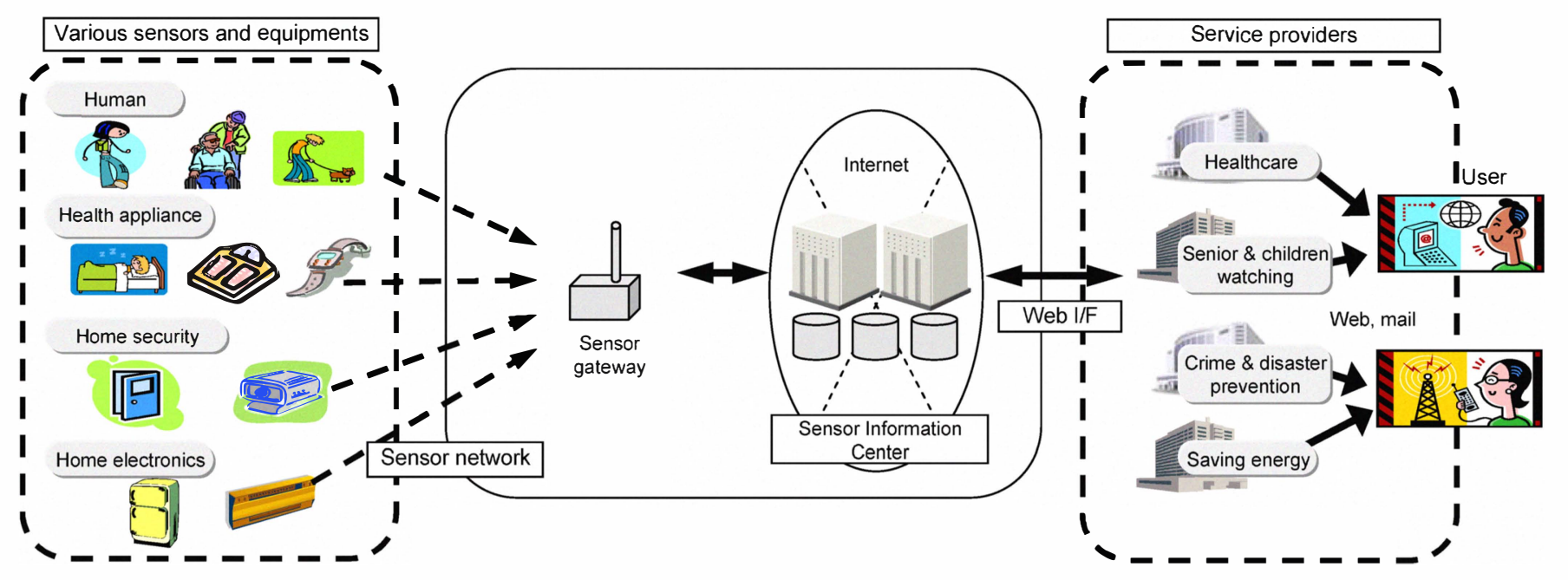

Fig. 1. Sensor Information Web Service

Section 2, and explain the sensor information web service system developed and describe a forthcoming evaluation study in Section 3. We analyze the questionnaire results collected from the participants of the present study in Section 4, and present our conclusions and considerations for future study in Section 5 .

\section{Outline of the Diabetes Prevention Program}

The diabetes prevention program called "Health Challenge Grand Strategy" has been run jointly by Osaka Prefecture University and Habikino city government since 2005. This enterprise is undertaken with the help of the Osaka insurer conference, with the School of Comprehensive Rehabilitation of Osaka Prefecture University and Habikino city government taking the lead. Cooperation is also provided by, for example, the Habikino Medical Association, the Habikino Dentists Association, and the Home Dietetic Association.

In 2008, the program was started with 53 participants and ran from June through November. Entry criteria to the program was having a $\mathrm{HbAl} 1 \mathrm{c}$ level between $5.2-6.1 \%$ and not receiving treatment for diabetes. It is considered that $\mathrm{HbAlc}$ reflects the

TABLE I

OUTLINE OF THE DIABETES PREVENTION PROGRAM

Orientation, Blood test, Body measurement
Test of physical strength, Exercise
Mouth check and Lecture on dental surgery
Lecture on dietary therapy, Body measurement
Lecture on oral care, Instruction on tooth brushing
Lecture on ideal body weight and required energy
Exercise
Lecture on "Prevention of a lifestyle-related diseases
Exercise, Instruction on tooth brushing
Training in cooking, Body measurement
Exercise, Instruction on tooth brushing
Assessment of nutrition, Body measurement
Test of physical strength
Oral Assessment
Closing session

Test of physical strength, Exercise

Mouth check and Lecture on dental surgery

Lecture on dietary therapy, Body measurement

Lecture on ideal body weight and required energy

Exercise

Exercise, Instruction on tooth brushing

Assessment of nutrition, Body measurement

Oral Assessment

15 Closing session

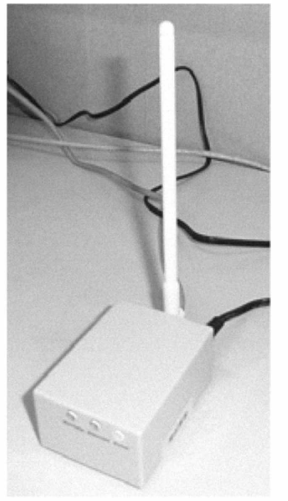

Fig. 2. A prototype of the sensor gateway

average blood sugar level for the past one to three months, and is therefore used as a marker for diagnosis as well as for ongoing status, and a level of not less than $5.2 \%$ is stated in specific health guidelines. The outline of the program's schedule in the current fiscal year is shown in Table I. The program is being carried out at Habikino Health Center and an Osaka Prefecture University Habikino campus facility.

\section{Diabetes Prevention Program Support Using A SENSOR Information Web SERVICE}

In recent years, with the miniaturization of communication devices and sensors, a home network can now be realized, and research and development involving highly computerized "smart homes" [11] is under way. The wireless communication system ZigBee is recognized as the standard technology for realizing such home networks. ZigBee offers a low-cost and a low-power consuming wireless system which is based on the IEEE 802.15.4 standard. It is possible to build home networks which carry out sensing of various information in the home environment through ZigBee. Various applications can be considered for home networks, including security, health 


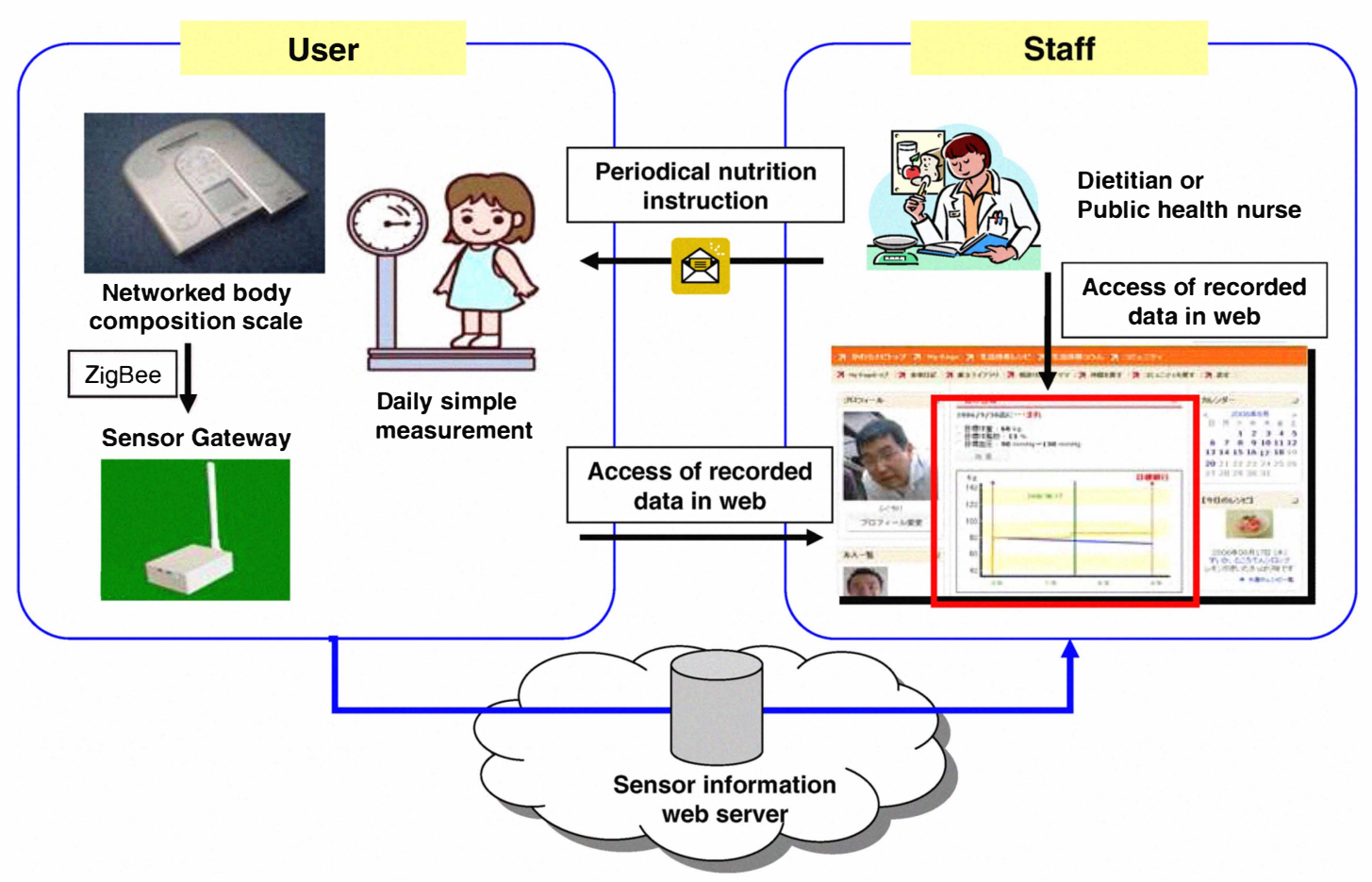

Fig. 3. Diabetes prevention program support using a sensor information web service

care support [12, 13] and home energy management systems (HEMS) [14].

The sensor information web service is considered a suitable means for realizing such a home network. The components of this service are shown in Fig. 1. The data obtained by various kinds of sensors and apparatus is transmitted through a wireless network based on ZigBee to the sensor gateway. A prototype of the sensor gateway is shown in Fig. 2. The gateway has a DHCP client function and setup is completed simply by accessing the Internet via a LAN cable. Since the system is very small, it takes up very little space at home. The sensor gateway transmits the collected data to the sensor information center via the Internet and the data is then stored on a server installed in the center. The sensor information accumulated at the center can be provided in various forms according to the demands of the application, or the needs of the service or end user.

Since data collection without the need to input data directly via a PC is made possible by using the sensor information web service, the burden on the user is eased dramatically when compared to the use a conventional data collection system which needs PC input. As shown in Fig. 3. The user need only stand on the body composition meter once a day. This means that there is no difference in the time and effort taken for usual self-management measurements when using the proposed system. The staff responsible for monitoring the participant's follow up will decide how the data is viewed or explained to the participant. For example, if the participant uses a PC, then a graph of weight measurements can be viewed via the Internet or a comment from staff can be read.
Moreover, not only sensor information can be collected during follow up, but also other health information such as diet and exercise can be input via the Internet. Input via a cellular phone is also possible. For those users who do not wish to or cannot use such communication devices, follow up can instead be done by telephone or mail with the data then being input by the staff responsible for follow up.

\section{QUESTIONNAIRE TO DETERMINE IMPRESSIONS OF Possible System Use by PROgRam Participants}

Using a questionnaire survey, we investigated the consciousness to use the proposed follow-up system among the 53 participants (11 men, 42 women; mean age [standard deviation] 63.6 years [4.6 years]) who had completed the diabetes prevention program, after first explaining to them the outline and the usage of the follow-up system based on the sensor information web service. We also investigated their Internet environment and current use of a PC or cellular phone. The results revealed that $71 \%$ of participants had a PC at home, although only $44 \%$ of participants had actual access to the Internet; $39 \%$ reported they could not use the Internet and $17 \%$ reported they were unsure whether they could use the Internet or not. The possession rate of a cellular phone was $67 \% ; 75 \%$ of these users had e-mail function on their cellular phone. However, none of the participants were using the web access function of their cellular phone.

The questionnaire results about the interest to the follow-up service are shown in Fig. 4. Although $31 \%$ of the program participants answered that they were interested in a followup system, only $19 \%$ answered that he or she would actually 
(a) Are you interested in follow-up service?

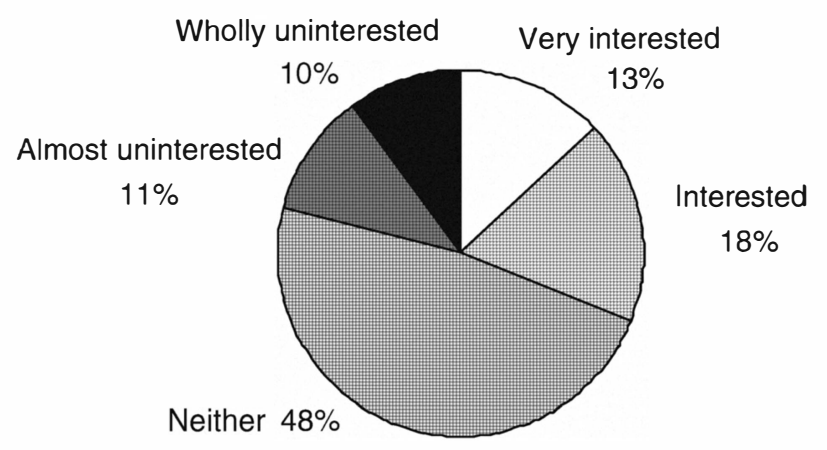

(b) Do you want to actually use follow-up service?

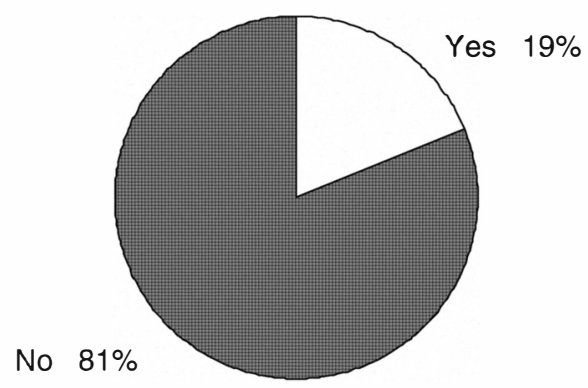

Fig. 4. Questionnaire results about the interest to the follow-up service

like to use one. The reasons given for not wishing to use the follow-up system are shown in Table II. As an advantage of the proposed system, the fact that a PC is not required for daily data collection was mentioned. However, most reasons given were "I do not use a PC." This response suggests that most elderly people believe the Internet is indivisible from a PC. Based on this finding, we consider that people who cannot use a PC will also believe they cannot use a system which uses only the Internet as the communication medium. Therefore, in order to disseminate the use of our proposed system, it is necessary to provide sufficient explanation during the diabetes prevention program to elderly participants in order to change any misunderstanding about system use.

Moreover, very few elderly people who use a PC and the Internet daily actually connect up the devices or set up a network themselves. In many cases, staff from a computer store, a network contractor or a young family member completes such tasks. The system we propose in this research needs to connect a gateway to the network in each house, but it is clearly difficult for many potential system users not to be able to complete this themselves or explain it sufficiently to family members. Even in the case of connecting only a LAN cable to begin operation, it is important that the system is explained directly to those who will complete the connection and set up the devices.
TABLE II

REASONS GIVEN FOR NOT WISHING TO USE THE FOLLOW-UP SYSTEM

I do not use a PC.

There is no internet environment in my home. 11

There is no PC in my home.

I can do self-control without follow-up service.

How to use the follow-up service seems to be difficult.

I am difficult to measure every day.

I cannot understand this service itself.

Other reasons

(Multiple answers are included)

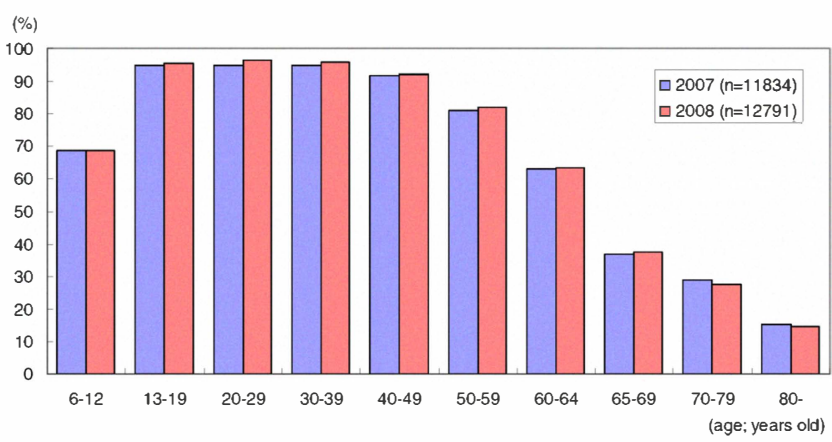

Fig. 5. Internet utilization rate by age in Japan (quoted from 2009 White Paper on Information and Communications in Japan)

\section{CONCLUSiONS AND Future WORK}

In this research, we proposed a health care support system using a sensor information web service based on ZigBee for the follow up of participants of a diabetes prevention program, and carried out a questionnaire survey among the participants about their awareness and understanding of follow up and impressions of potential use of the system. It became clear that a number of obstacles concerning the living environment of elderly people need to be addressed to ensure suitable implementation of the proposed system. The solutions we consider necessary are as follows.

1) The participants themselves could learn the skills required to perform the set up of the devices. Such training could be provided in a lecture specifically on how to introduce the system at home. However, we note that many elderly people are not interested in the Internet or PC (Fig. 5) and may have limited interest in such a lecture and motivation to attend might be low.

2) The Internetwork environment currently available in the user's home would not used by the system but instead a gateway would connect to the Internet via a mobile access router. Alternatively, the gateway itself would have a public wireless or $3 \mathrm{G}$ connection function. A system improvement of this kind would make the introduction of this system easy and the user could begin using the system immediately only by taking the devices home. However, in this case, there is the drawback of a cost of a telecommunication line associated with such usage.

3) The proposed follow-up system could be introduced not 
as an independent service but as a part of a service to elderly people using a full sensor information web service. In other words, we would position the followup system as an additional service to the home in which the gateway is already installed in order to use other applications, such as energy saving and security applications. However, in this case, there is a drawback in that those who can use this system will be limited.

Irrespective of the solutions posited above, the most important concern for users is the starting and ongoing expense of system usage $[15,16]$. From an economical viewpoint, we are now examining what kind of method would be most suitable for making the system used in a widespread manner. We are also examining a concrete method of providing dietary advice to system users, aiming at clarifying the content and frequency of advice which will be effective for successful follow-up.

\section{REFERENCES}

[1] R. Sakamoto, K. Watanabe, A. Yazawa, S. Imai, and M. Imaki, "Characteristic physical activity and fitness of women participating in a diabetes prevention class in relation to the degree of obesity," Jpn. J. Health Fit. Nutr., vol. 13, no. 1, pp. 38-43, 2008.

[2] K. Watanabe, A. Yazawa, K. Watanabe, S. Imai, R. Sakamoto, and M. Imaki, "Changes in extremity muscle mass of obese middle-aged and elderly women with body wight loss," Health and Behavior Science, vol. 6, no. 1, pp. 21-26, 2007.

[3] A. Yazawa, K. Watanabe, Y. Yoshida, M. Imaki, and S. Tanaka, "Effects on middle-age and elderly people on intervention with a health promotion program using water exercise: Evaluation of physical activity, muscle mass, physical fitness and blood test value," J. Anal. Bio-Sci., vol. 30, no. 3, pp. 271-278, 2007.

[4] _ , "Relationship between physical activity measured by accelerometer and muscle mass, physical fitness and blood test values in middleaged and elderly people," J. Anal. Bio-Sci., vol. 30, no. 3, pp. 279-285, 2007.

[5] A. Yazawa, K. Watanabe, A. Saruwatari, and M. Imaki, "Relationship between extremity muscle mass and physical fitness in obase middleaged and elderly women," Jpn. J. Health Fit. Nutr., vol. 11, no. 2, pp. 3-9, 2006.

[6] S. Yamaguchi, "Cause of weight rebound with obese women who had lost weight and effect of intervention by telephone and newsletters," Jpn. J. Nutr. Diet., vol. 65, no. 1, pp. 21-28, 2007.

[7] L. Mamykina and E. D. Mynatt, "Investigating and supporting health management practices of individuals with diabetes," in Proceedings of the 1st International Workshop on Systems and Networking Support for Healthcare and Assisted Living Environments (HealthNet '07), Jun. 2007, pp. 49-54.

[8] A. R. Jadad and T. Delamothe, "What next for electric communication and health care?" BMJ, vol. 328, pp. 1143-1144, May 2004.

[9] M. Sugano, R. Araki, and N. Aiba, "Evaluation of a web-based selflearning system for lifestyle improvement," in Proceedings of the 1st International Workshop on Connectivity, Mobility and Patients' Comfort, Jan. 2008.

[10] ZigBee Alliance, "ZigBee Specification, r17," Oct. 2007.

[11] X. Carcelle, B. Heile, C. Chatellier, and P. Pailler, "Next WSN applications using ZigBee," in Proceedings of IFIP Home Networking Conference, Dec. 2007, pp. 313-328.

[12] P. Fergus, D. Llewellyn-Jones, M. Merabti, and A. Haroon, "Networked appliances for home healthcare and lifestyle management," in Proceedings of IFIP Home Networking Conference, Dec. 2007, pp. 313-328.

[13] S. Dagtas, Y. Natchetoi, H. Wu, and A. Shapiro, "An integrated wireless sensing and mobile processing architecture for assisted living and healthcare applications," in Proceedings of the 1st International Workshop on Systems and Networking Support for Healthcare and Assisted Living Environments (HealthNet '07), Jun. 2007, pp. 70-72.
[14] N. Kushiro, S. Suzuki, M. Nakata, H. Takahara, and M. Inoue, "Integrated residential gateway controller for home energy management system," IEEE Transactions on Consumer Electronics, vol. 49, no. 3, pp. 629-636, Aug. 2003.

[15] M. Cocosila, N. Archer, and Y. Yuan, "Would people pay for text messaging health reminders?" Telemedicine and e-Health, vol. 14, no. 10, pp. 1091-1095, Dec. 2008.

[16] M. E. Davalos, M. T. French, A. E. Burdick, and S. C. Simmons, "Economic evaluation of telemedicine: Review of the literature and research guidelines for benefit-cost analysis," Telemedicine and e-Health, vol. 15, no. 10, pp. 933-948, Dec. 2009. 